personal goals can help physicians providing targeted and personalised medicine.

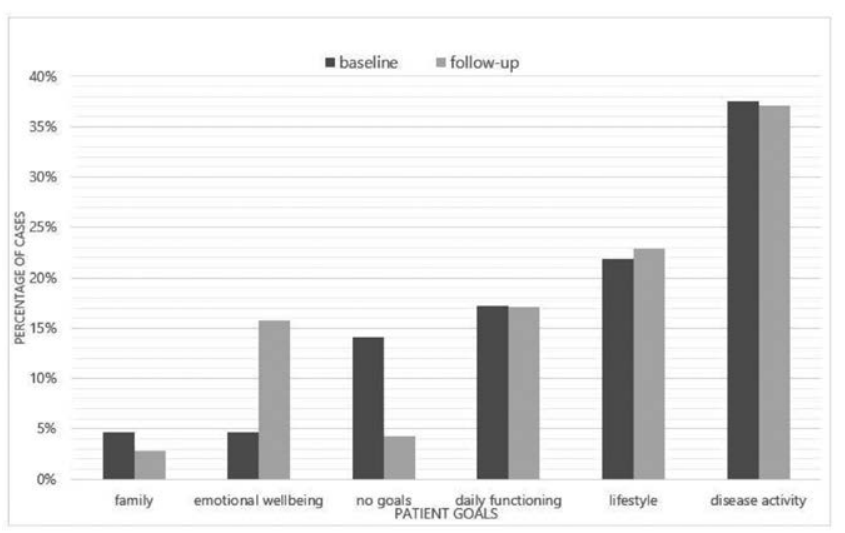

Figure 1. themes of treatment goals at baseline and follow-up

Acknowledgements: The researchers would like to thank all patients participating in this study and acknowledge the UMC Utrecht research program Infection and Immunity for supporting this initiative.

Disclosure of Interests: None declared

DOI: 10.1136/annrheumdis-2021-eular.259

\section{AB0885-HPR SLEEP IN PATIENTS WITH RHEUMATOID ARTHRITIS}

C. Andersen ${ }^{1}$, K. M. Latocha2, B. A. Esbensen ${ }^{1} .{ }^{1}$ Rigshospitalet, Copenhagen Center for Arthritis Research (COPECARE), Center for Rheumatology and Spine Diseases, Glostrup, Denmark; ${ }^{1}$ Rigshospitalet, Copenhagen Center for Arthritis Research (COPECARE), Center for Rheumatology and Spine Diseases, Glostrup, Denmark

Background: Many patients with rheumatoid arthritis (RA) live with the consequences of arthritis in everyday life. Sleep disturbances, including insomnia, are highly prevalent and a complex issue that increases existing RA-related symptoms, such as pain, fatigue, and depressed mood. To our knowledge, only two studies have qualitatively investigated the patient perspective and experiences of sleep in people with RA (1-2).

Objectives: To investigate how people with RA and concomitant sleep disturbances describe their sleep and experience everyday life with poor sleep. Methods: This study is a qualitative phenomenological study. In total, 14 people with RA and sleep disturbance were recruited from an RCT study (3) from the Center for Rheumatology and Spine Diseases at Rigshospitalet in 2012. Semi-structured interviews were performed after the last follow-up in the RCT. The analysis was based on Giorgis' descriptive phenomenological psychological analysis.

Results: For the characteristics of participants, see Table 1. We identified six essences; 1) "When sleep is put into words" covered that the participants described their poor sleep experiences simultaneously to their wishes for good sleep still existed. 2) "The struggle to sleep" included participants' descriptions of difficulties sleeping and how thoughts and worries could interfere with sleep at night. 3) "Pain is a companion day and night" included how pain affected sleep and how participants had problems with pain management. 4) "To take sleep in their own hands" described how the participants tried to create their framework for sleeping and how they also strove to reach out for help from, e.g. of professionals. 5) "Everyday life in the shadow of sleep" included the overwhelming fatigue, a need to rest during the day, and how social relationships were affected negatively. 6) "Adapt to the circumstances," which meant that the participants tried to seek shelter behind a facade, and at the same time tried to learn to live with the consequences of poor sleep in everyday life.

People with RA and sleep disturbances describe several challenges that affect their sleep, e.g., pain, thoughts at night, and many reasons for awakenings. Participants in this qualitative study were not aware of the difference between fatigue and poor sleep, both regarding reasons for occurring and management of the two highly prevalent consequences of RA. At the same time, they believed that health professionals lacked knowledge on sleep disturbances and management hereof in people with RA.

Conclusion: When people with RA and concomitant sleep disturbance describe their sleep, a struggle to sleep was most often described, characterized by pain, thoughts, difficulty falling asleep, and many and early awakenings. Everyday life experience with sleep disturbances was marked by fatigue and sleep deprivation, negative impact on social skills and relationships, and failure when they reached out for help.

Table 1. Characteristics of participants

\begin{tabular}{|c|c|}
\hline & $(\mathrm{N}=8)$ \\
\hline Age, median (range) & $50(44-60)$ \\
\hline Sex, Women, $\mathrm{n} \%$ & $7(77,8 \%)$ \\
\hline \multicolumn{2}{|l|}{ RA-related } \\
\hline Duration of RA, median (range) & $14,75(6-32)$ \\
\hline DAS28-CRP, median (range) ${ }^{1}$ & $1,9(1,62-3,03)$ \\
\hline Physical function, $\mathrm{HAQ}$, median (range) ${ }^{1}$ & $0,5(0,000-2,13)$ \\
\hline \multicolumn{2}{|l|}{ Sleep } \\
\hline PSQI, median (range) ${ }^{2}$ & $9,5(6-15)$ \\
\hline \multicolumn{2}{|l|}{ Fatigue } \\
\hline VAS fatigue, median (range) & $33,4(2-65)$ \\
\hline BRAF-MDQ, median (range) ${ }^{3}$ & $25,5(4-36)$ \\
\hline \multicolumn{2}{|l|}{ Pain } \\
\hline VAS pain, median (range) & $25(0-60)$ \\
\hline \multicolumn{2}{|l|}{ Quality of Life } \\
\hline VAS global, median (range) & $45,6(8-40)$ \\
\hline
\end{tabular}

Disease activity score-28 C-reactive protein ${ }^{2}$ Health Assessment Questionnaire, score 0-3 (Higher score indicates worse physical functioning. ${ }^{2}$ Pittsburgh Sleep Quality Index, score $0-21$ (score of 5 or more indicates sleep disturbances) ${ }^{3}$ Visual Analog scale, score 0-100 (higher score is worse) ${ }^{4}$ Bristol Rheumatoid Arthritis Fatigue Multidimensional Questionnaire score 0-70(higher score indicates more fatigue)

\section{REFERENCES:}

[1] Short V et al; Annals of the Rheumatic Diseases. 2017;76(Suppl 2):519.

[2] McKenna S, et al; EULAR. 2020; OP0267-HPR (2020).

[3] Løppenthin et al; BMC Musculoskeletal Disorders 2014, 15:49.

Disclosure of Interests: Camilla Andersen: None declared, Kristine Marie Latocha: None declared, Bente Appel Esbensen Speakers bureau: Bente Appel Esbensen has received speaking fees from Pfizer and Eli Lilly. DOI: 10.1136/annrheumdis-2021-eular.332

\section{AB0886-HPR ASSESSMENT OF SEASONAL VARIATIONS ON CHRONIC INFLAMMATORY RHEUMATISMS ACTIVITY}

O. Hamdi ${ }^{1}$, M. Sellami ${ }^{1}$, S. Miladi ${ }^{1}$, A. Fazaa ${ }^{1}$, L. Souabni ${ }^{1}$, K. Ouenniche ${ }^{1}$, S. Kassab ${ }^{1}$, S. Chekili ${ }^{1}$, K. Ben Abdelghani ${ }^{1}$, A. Laatar ${ }^{1} .{ }^{1}$ Mongi Slim Hospital, Rheumatology, Tunisia, Tunisia

Background: Although rheumatoid arthritis (RA) and spondyloarthritis (SA) activities have been described to vary under the influence of several factors, little is known about the influence of seasonality on the activity of chronic inflammatory rheumatisms.

Objectives: To assess the influence of seasonality on the activity of chronic inflammatory rheumatisms.

Methods: We conducted a cross-sectional study involving patients with RA (2010 ACR/EULAR criteria) and SA (2009 ASAS criteria). Chronic inflammatory rheumatisms activity was assessed during the summer (June-September) and winter (December-February) using clinical parameters including the Patient's Global Assessment of disease activity (PGA), 10-cm Visual Analog Scale (VAS) pain score, morning stiffness, nocturnal awakenings, and joint count for RA (tender joint count (TJC) and swollen joint count (SJC)); biological parameters including erythrocyte sedimentation rate (ESR) and C-reactive protein (CRP); disease activity scores including the SDAI, CDAI and DAS for RA, BASDAI and ASDAS ${ }_{\text {CRP }}$ for SA. An analysis of variance (ANOVA) was used to assess the statistical relationship between seasonality and rheumatisms activities.

Results: We enrolled 175 patients (100 RA and $75 \mathrm{SA}$ ) with a sex ratio of 0.5 and a mean age of $57.75 \pm 10.53$ years [23-83]. The mean chronic inflammatory rheumatisms duration was $12.38 \pm 4.6$ years. RA was erosive in $91 \%$ of cases. Rheumatoid factor and anti-citrullinated peptides antibodies were positive respectively in $84 \%$ and $85 \%$ of cases. Seventy-five percent of RA patients were on corticosteroids with a mean dose of $10.14 \mathrm{mg} /$ day of prednisone equivalent and $79 \%$ of SA patients were on non-steroidal anti-inflammatory drugs. Eighty percent of our patients were treated with conventional synthetic DMARD and $44 \%$ with biological DMARD. Small joints were more affected than large joints regardless of the season in RA patients $(p=0.05)$. The following parameters were higher in winter than in summer in RA patients: mean PGA 4.73 vs $4.64(p=0.01)$; mean morning stiffness 1.6 vs $1.1 \quad(p=0.01)$; mean SJC 8.7 vs $7.5(p=0.01)$; mean DAS ${ }_{28}$ ESR 4.56 vs $3.99(p=0.05)$; mean DAS28 CRP 4.6 vs $3.41(p=0.05)$, mean SDAI 21.8 vs $19.5(p=0.05)$; mean CDAI 20.5 vs $18.75(p=0.01)$ and mean ESR $45.6 \mathrm{~mm} / \mathrm{h}$ vs $38.2 \mathrm{~mm} / \mathrm{h}(p=0.01)$. As for $S A$, the following parameters were higher in winter than in summer: mean morning stiffness 2 vs $1.4(p=0.01)$; mean ASDASCRP 3.9 vs $3.1(p=0.01)$ and mean BASDAI 6.2 vs $4.9(p=0.05)$. However, we found no statistically significant 
correlation between seasonal changes and VAS pain score, nocturnal awakenings, TJC, and CRP.

Conclusion: Chronic inflammatory rheumatisms activity was higher in winter. Health care professionals should take seasonal changes into account in order to improve therapeutic care.

Disclosure of Interests: None declared

DOI: 10.1136/annrheumdis-2021-eular.412

\section{AB0887-HPR CHARLSON COMORBIDITY INDEX (CCI) IN RHEUMATOID ARTHRITIS: CLASSIFICATION AND CORRELATIONS}

C. Flourou ${ }^{1}$, S. Psarelis ${ }^{2}$, A. Tofarides ${ }^{3}$, E. Papanicolaou4, G. Papazisis ${ }^{5}$. ${ }^{1}$ Nicosia General Hospital, Internal Medicine Department, Nicosia, Cyprus; ${ }^{2}$ Nicosia General Hospital, Rheumatology Department, Nicosia, Cyprus; ${ }^{3}$ Nicosia General Hospital, Internal Medicine Department, Nicosia, Cyprus ${ }^{1}$ Nicosia General Hospital, Internal Medicine Department, Nicosia, Cyprus: ${ }^{5}$ Aristotle University of Thessaloniki, Clinical Pharmacology, Thessaloniki, Greece

Background: Charlson Comorbidity Index[1] is a tool including age and chronic diseases assessing the comorbidity burden. The age and the comorbidity burden in RA patients determine the morbidity and mortality.

Objectives: To assess and classification of $\mathrm{CCl}$ in RA patients with usage of the health-care system (outpatient clinics) in a real-world setting.

Methods: 327 patients with RA from a large outpatient service of a central hospital were retrospectively reviewed. Demographic characteristics, treatment for RA and comorbidities were recorded. Charlson Comorbidity Index (CCl) was measured and classified as low, intermediate and high score for 1-2, 3-4 and $>=5$ points, respectively. Its correlation with polypharmacy and necessity of biologic DMARDs was studied. Univariable and multivariable analyses were performed.

Results: Data from 327 RA patients (75,8\% females, $24,2 \%$ males) with a mean $\pm S D$ age of $63 \pm 11,8$ years and disease duration $113 \pm 63$ months, were recorded.

$\mathrm{CCl}$ was $3 \pm 1,2$ points (mean $\pm \mathrm{SD}$ ) and maximum score was observed at 7 points. High score ( $>=5$ points) was observed at $9,2 \%$ and in the majority the score was intermediate (3-4points) at $55 \%$. All the RA patients with high score fulfilled the criteria of polypharmacy. Patients with high score had 9,7 times more probability of polypharmacy than the patients with low score $(p=0.09$, $1.4-2.595 \% \mathrm{Cl}$ ).

70 patients were treated with biologic-DMARDs $(21,7 \%)$, in the majority with TNFa inhibitors $(16,5 \%)$. In RA patients receiving biologic-DMARDs was observed low or intermediate score of $\mathrm{CCl}$. The most likely explanation is the severity of the disease that predominated, its complications and the possible overlap with other conditions.

Conclusion: The majority of RA patients had intermediate score of $\mathrm{CCl}$. In patients with high score-meaning more comorbidities- polypharmacy was observed completely. Patients receiving biologic-DMARDs characterized with less comorbidities.

REFERENCES:

[1] Charlson E M et al. A new method of classifying prognostic comorbidity in longitudinal studies:development and validation. J Chronic Dis. 1987;40(5):373-83

Disclosure of Interests: None declared

DOI: 10.1136/annrheumdis-2021-eular.1103

\section{AB0888-HPR EULAR SJOGREN'S SYNDROME PATIENT REPORTED INDEX-FATIGUE SCORE AND HADS-DEPRESSION SCORE MEDIATE IDENTITY SCORE OF ILLNESS PERCEPTION QUESTIONNAIRE IN PATIENTS WITH PRIMARY SJÖGREN'S SYNDROME}

A. Kapusuz ${ }^{1}$, K. Abacar ${ }^{2}$, Y. Yenisoy ${ }^{1}$, İ. Tatı ${ }^{3}$, E. N. Çakır ${ }^{1}$, F. Türe-Özdemir ${ }^{3}$, M. Yay ${ }^{4}$, Ü. Karaçaylı $I^{5}$, H. Direskeneli ${ }^{2}$, N. Inanc ${ }^{2}$, G. Mumcu ${ }^{6}{ }^{1}$ Marmara University, Institute of Health Sciences, Istanbul, Turkey; ${ }^{2}$ Marmara University Medical School, Department of Rheumatology, Istanbul, Turkey; ${ }^{3}$ Marmara University Medical School, Department of Heamatology and Immunology, Istanbul, Turkey; ${ }^{4}$ Mimar Sinan Fine Arts University, Department of Statistics, Istanbul, Turkey; ${ }^{5} \mathrm{Health}$ Sciences University, Department of Oral and Dental Surgery, Istanbul, Turkey; ${ }^{6}$ Marmara University, Department of Health Management, Istanbul, Turkey

Background: Dryness, fatigue, and pain are common clinical manifestations assessed by EULAR Sjogren's Syndrome Patient Reported Index (ESSPRI)-Dryness, -Fatigue, -Pain scores in patients with primary Sjögren's syndrome (pSS). In addition, depression is also seen in these patients owing to the pattern of the chronic disease.
Objectives: The aim of the study was to assess the complex interactions among Depression status, Illness Perception, and prominent clinical manifestations evaluated by the ESSPRI (Dryness, Fatigue, and Pain) in patients with pSS.

Methods: In this cross-sectional study, 111 patients with pSS (M/F: 5/106; mean age: $52.9 \pm 12.01$ years) were included. The data were collected by clinical examination and a questionnaire regarding patient reported outcome measures (PROMs). Unstimulated (U-WSFR) and stimulated (S-WSFR) whole saliva flow rates of patients were calculated as $\mathrm{ml} / \mathrm{min}$. Hospital Anxiety and Depression Scale (HADS), Illness Perception Questionnaire-R (IPQ-R) and EULAR Sjogren's Syndrome Patient Reported Index were filled by patients. Increases in HADS score and subgroup scores of ESSPRI (Dryness, Fatigue and Pain) and IPQ-R dimensions regarding Identity, Consequences, and Emotional reflected poor conditions for patients. In addition, patients scored their disease activity ( 0 : inactive-100: the worst activity) by using 100-mm visual analogue scale (VAS) After preliminary analysis, a mediation analysis was used to evaluate the relations among these variables.

Results: In the study, ESSPRI-Dryness score $(6,27 \pm 2,79)$ was associated with U-WSFR $(0,40 \pm 0,57)$ and S-WSFR $(1,04 \pm 0,86),(r:-0,4 p=0.000 ; r:-0,3 p=0.004)$ Moreover, patients reported disease activity score $(48,78 \pm 26,67)$ was related to U-WSFR ( $r:-0,3 p=0.026)$ as well as Consequence $(19,12 \pm 5,47)$ and Emotional $(19,54 \pm 7,02)$ scores of IPQ-R questionnaire ( $r: 0,3 p=0.035 ; r: 0,3 p=0.014)$. In IPQ-R questionnaire, Identity score $(8,04 \pm 3,1)$ reflecting number of symptoms that patients experienced due to their illness was correlated with scores of ESSPRI-Fatigue $(5,29 \pm 2,97)$, ESSPRI-Pain $(5,18 \pm 3,01)$, HADS-Anxiety $(11,67 \pm 5,55)$, HADS-Depression $(9,2 \pm 4,98)$ in the study $(p<0.05)$.

In the mediation analysis, Identity score was directly mediated by ESSPRI-Fatigue score $(p=0.0093)$ and indirectly mediated by HADS-Depression score $(p=0.0011)$

A bootstrap analysis with 5000 replications was applied to estimate mediation effect to generate $95 \% \mathrm{Cl}$. Percentile bootstrap of HADS-Depression was found to be an effective mediator for Identity score based on 5000 bootstrap sample. Conclusion: Both depression status and fatigue affected Identity score reflecting the number of symptoms poorly. Considering this complex relationship in disease activity assessment may positively affect disease outcomes.

Disclosure of Interests: None declared

DOI: 10.1136/annrheumdis-2021-eular.1893

\section{AB0889-HPR PERCEIVED SATISFACTION WITH CHRONIC PAIN CARE IN GERMAN PATIENTS WITH FIBROMYALGIA (FM)}

M. Offenbächer ${ }^{1}$, L. Toussaint ${ }^{2}$, J. Hirsch ${ }^{3}$, M. Weigl ${ }^{4}$, N. Kohls ${ }^{5}$, M. Vallejo ${ }^{6}$, J. Rivera ${ }^{7}$, F. Sirois ${ }^{8}$, J. Dezutter ${ }^{9}{ }^{1}$ Gasteiner Heilstollen, Medical Department, Bad Gastein, Austria; ${ }^{2}$ Luther College, Psychology, Decorah, United States of America; ${ }^{3}$ East Tennessee State University, Psychology, Johnson City, United States of America; ${ }^{4}$ Klinikum Großhadern, Department of Orthopaedics, Physical Medicine and Rehabilitation, München, Germany; ${ }^{5}$ Coburg University of Applied Sciences, Health Promotion, Coburg, Germany; ${ }^{6}$ UNED, Psychology, Madrid, Spain; ${ }^{7}$ Gregorio Marañón Hospital, Rheumatology, Madrid, Spain; ${ }^{8}$ The University of Sheffield, Psychology, Sheffield, United Kingdom; ${ }^{9}$ University of Leuven, School Psychology and Development in Context, Leuven, Belgium

Background: In chronic pain care a multidimensional perspective with attention to patients' cognitions, emotions, and their ability to cope is needed (1) Previous studies are also pointing to the role of experiencing meaningfulness in life in the adjustment to disability. Therefore care should additionally focus on the existential domain of patients' lives to live up to a holistic care approach (2). However, there are only a few studies on how FM patients are satisfied with practitioners' attention to multiple aspects of life with a chronic pain condition. Objectives: To assess perceived satisfaction with chronic pain care and its associations with health variables in a cohort of patients with FM.

Methods: We invited 18 FM self-help groups in Germany to participate anonymously in our survey and sent them in total 192 paper-and-pencil surveys Sociodemographics, disease related variables (e.g. pain, general health) and psychological variables [e.g. depression, anxiety, hope, stress] were assessed with standardized instruments, including 5 items (answer format $1=$ very unsatisfied $-10=$ very satisfied) assessing subjective satisfaction with medical care in different domains with the following questions: How satisfied are you with the attention of your treatment team/physician at home for physiological aspects of your pain (Physio)/ the consequences of the pain on your physical functioning (Physical)/ on your psychological well-being (Mental)/ on your social life (Social)/ on your meaning in life (Meaning).

Results: In total $162 \mathrm{FM}$ patients participated ( $=84 \%$ response rate). Their mean age was 58 years $(S D=10), 84 \%(N=135)$ were female. Highest level of education was: Elementary School 29\%, Junior High School 35\%, High School $15 \%$, College $12 \%$, and other $10 \%$. Duration of chronic pain was 